\section{Object Index}

$0108+388112$

0109-383 72

$0149+710176$

$0202+149116$

$0218+357 \quad 94$

$0219+16480$

$0235+16480$

0405-385 84, 90, 92, 475

0420-014 106

0440-033 116

$0446+112116$

$0502+67580$

$0524+034100$

$0548+165138$

0637-752 118

$0710+439112$

$0716+714 \quad 80,96$

$0735+178 \quad 134$

$0827+243 \quad 116$

$0829+04680,116$

$0836+710126$

$0917+62484$

$0954+556 \quad 116$

$0954+658 \quad 80,106$

$0957+561 \quad 346$

$1028+51180$

$1156+29580$

$1159+291102$

$1219+28580$

1229-021 116

$1331+170 \quad 116$

1334-127 134

1351-017 118

1510-089 116, 142

$1512+36 \quad 166$

1519-273 84, 92

1524-136 138

$1604+159116$

$1606+106 \quad 116$

$1636-536 \quad 244$

$1803+784118,134$

$1807+698 \quad 176$

$1819+384584,88,475$

1908-201 116

$1915+1052$

1921-293 128

$1946+708182,194$
$1928+783118$

$1959+650 \quad 114$

$1928+783118$

2022-077 116

2155-304 114

$2209+236 \quad 116$

$2215+020118$

$2250+194318$

$2251+158 \quad 318$

2322-123 182,196

$2344+514114$

$2352+495 \quad 112$

$2356+196 \quad 116$

$41.95+575208,376$

$43.31+592366,374$

1E 0102.2-7219 358

3C $48 \quad 162$

3C 6680,114

3C 8448,118182

3C $216 \quad 190$

3C 234190

3C $236 \quad 182$

3C 27980,118

3C $293 \quad 182$

$3 \mathrm{C} 345 \quad 130$

3C 390.376

3C 391398

4C $31.04 \quad 182$

IZw $1340,344,475$

IZw 18222

II Zw 40224

III Zw 2110

III Zw $35 \quad 326$

Antares 316

AqlX-1 244

Arches Cluster 276

Arp 220 182, 326, 366, 376, 386, 475

Arp 299198

Betelgeuse 288, 316, 412

BL Lac 80,82

BR 1202-07 432

Breysacher $73 \quad 312$

Capella 457

Cas A 358,366

Centaurus A $66,182,194$

Circinus 182, 334

CrabNebula 10

CXOJ004244.2+411609 38

CXOUJ09550.2+694047 208 
Cygnus A 10, 182

CygnusLoop 398

DQ Her 260

FH Ser 260

FIRST $031 \quad 102$

G21.5-0.9 358

G266.2-1.2 358

$\mathrm{G} 292.0+1.8 \quad 358$

G347.3-0.5 358

G348.5+0.1 398

G349.7 398

GalacticCenter $10,36,356$

GRO J1655-40 244

GRS 1915+105 2, 244

HB9 398

HD $32228 \quad 312$

HDF $850.1 \quad 102$

HFF $3642+1331 \quad 102$

HH 211236

HH 212236

HM Sge 306

Hydra A 182

IC 342154

IC $348 \quad 236$

IC 443398

IC $694182,198,236$

IC 4553326

IC 5063182

IM Peg 318

IR 17208-0014 326

IR $18580+6527 \quad 172$

IRAS 4A 252

IRAS 4B 252

IRAS $04000+5052284$

IRAS 05413-0104 252

IRAS 16253-2422 252

IRAS $20126+4104280$

IRC+10216 296, 310

Kes 67398

LargeMagellanic Cloud 366, 398

$\mathrm{LSI}+61^{\circ} 303 \quad 270$

M31 38, 200, 202, 352, 366

M33 366, 398

M66 390

M81 42, 380, 384, 390

M82 202, 208, 366, 374, 376, 378, 412,475

M83 224

M87 118136
M100 390

MCG-6-30-16 2

MilkyWay 234, 398

Mizar A 300

Mrk171 198

Mrk231 70, 182, 212, 326

Mrk273 182, 212, 326, 366

Mrk348 70

Mrk421 80, 114

Mrk501 114, 118

Mrk926 72

NCG 19362

NGC 253 202, 224, 336, 400

NGC 315154

NGC 38362

NGC 470228

NGC 661178

NGC 1023200

NGC 1052182,326

NGC $106870,72,182,216,326,334$, 338,340

NGC $127548,118,182$

NGC 1333252

NGC 1643394

NGC $1637 \quad 390$

NGC 1569222

NGC 2044312

NGC 211072

NGC $2146 \quad 180,366$

NGC 2363222

NGC 263970

NGC 269458

NGC 2782228

NGC 3079196,326

NGC $3227 \quad 182$

NGC 3256208

NGC 331058

NGC 3351228

NGC $3448 \quad 366$

NGC 3504228

NGC 3606236

NGC $3690198,326,394$

NGC $3877 \quad 390$

NGC 3894194

NGC $3953 \quad 350$

NGC 3994182

NGC 399850

NGC $4038202,206,366$

NGC $4039202,206,366$ 
NGC 404158

NGC 4102228

NGC $4151 \quad 168,182,190$

NGC 4214222

NGC $42582,182,326,334,338,475$

NGC $426144,182,190$

NGC 4314228

NGC 438872

NGC 4449154

NGC 4536228

NGC 4569228

NGC 4736366

NGC 4945182,326

NGC 525272

NGC 5253222,224

NGC 550670

NGC 554878

NGC $5793 \quad 326$

NGC 6240154,220

NGC 625174,144

NGC 6509154

NGC $6946 \quad 390$

NGC 6951228

NGC 705274,154

NGC 7331200

NGC $7469 \quad 340$

OA 184398

OI 09080

OJ 28780,134

ON 23180

OrionKL 258

Orionproplyds 272,274

OQ 24080

PSR 0329+54 404

PSR 0540-69 358

PSR 1534+12 408, 410

PSR B1913+16 408, 410

PSR 1929+10 404

PSR 2021+51 404

$\mathrm{R}$ Cas 322

R Doradus 298

RR Pic 260

R 5398

R 136224

RCW 38236

RSN 1986J 376

SAX J1808-3658 244

Sco OB2 404

SGR 0529-66 244
SGR 1627-41 244

SGR 1802-23 244

SGR 1806-20 244

SGR 1900+14 244

$\mathrm{SgrA}^{*} \quad 20,28,32,92,475$

SN 1006358,398

SN 1979C 390

SN 1980K 390

SN 1987A 358,396

SN 1993J $380,384,390$

SN 1997bs 390

SN 1998S 390

SN 1998T 394

SN $1999 \mathrm{em} 390$

SN 1999et 394

SN $2000 \mathrm{~cm} 394$

SNR 0540-6944 358

S Per 316

SS 433266,268

SV 513256

Taurus-Auriga 236

T Cep 288

Trapezium 236

TX Cam 252, 475

UGC 5456222

UrsaMajor Cluster 350

VW Cep 314

VY CMa 316

V533 Her 260

V705 Cas 260

V723 Cas 260

W 28398

W 44398

WB89 $520 \quad 284$

$\delta$ Cep 300

$\mu$ Cep 316

$\chi$ Cyg 288

$\zeta$ Oph 404

$\zeta$ Ori 300 